\title{
Diagnostic de l'Etat de Fertilité des Sols Sous Culture Cotonnière Dans les Principaux Bassins de Production de Côte d'Ivoire
}

\section{Emmanuel N'Goran Kouadio, PhD}

Centre National de Recherche Agronomique (CNRA)/ Département Agronomie, Station de recherche sur le Coton Bouaké, Côte d'Ivoire

\section{Emmanuel Kassin Koffi, PhD}

Centre National de Recherche Agronomique (CNRA)/ Laboratoire Central Sols Eaux et Plantes, Bouaké, Côte d'Ivoire

\section{Kouakou Brou Julien, PhD}

Centre National de Recherche Agronomique (CNRA)/ Département Technologie Coton graine, Station de recherche sur le Coton Bouaké,

Côte d'Ivoire

\section{Gustave Francis Messoum, PhD}

Ministère de l'Enseignement Supérieur et de la Recherche Scientifique Direction de la Programmation de la recherche

Kouamé Brou, PhD

Dominique Brou N'guessan,

Centre National de Recherche Agronomique (CNRA)/

Laboratoire Central Sols Eaux et Plantes, Bouaké, Côte d'Ivoire

\section{Doi: 10.19044/esj.2018.v14n33p221 URL:http://dx.doi.org/10.19044/esj.2018.v14n33p221}

\begin{abstract}
The study was conducted to assess the fertility potential of cotton soils in Côte d'Ivoire. A total of 500 plots of cotton farmers in 5 regions of the cotton basin was selected for soil sampling in the horizon $0-20 \mathrm{~cm}$. The chemical analyzes of soil samples in the laboratory were performed on the following parameters: particle size, $\mathrm{pHwater}$, organic matter, total $\mathrm{N}$, available $\mathrm{P}, \mathrm{K}, \mathrm{Ca}, \mathrm{Mg}$ and Cation Exchange Capacity (CEC) of soil. The physical characterization revealed that the studied soils are mainly sandy texture in the surface horizon. This gives them a very low retention capacity. The chemical characterization of soils showed that they have reactions ranging from slightly acid to neutral $(6.4<\mathrm{pH}<7)$. These soils have very low levels of total organic matter, total nitrogen, available phosphorus (in northern, south-central and western regions), calcium, potassium, and low values of cation exchange capacity.
\end{abstract}


Keywords: Cotton, Soil, Fertility, Productivity, Côte d'Ivoire

\section{Resumé}

La fertilité des sols est un paramètre majeur dans la productivité des cultures. En zone tropicale, cet état du sol est soumis à de nombreuses contraintes qui impactent négativement les rendements, en absence de fertilisation. C'est le cas du coton, en Côte d'Ivoire dont les rendements sont jugés faibles. Ils sont de l'ordre de $1400 \mathrm{~kg} / \mathrm{ha}$. La présente étude a été réalisée dans le but d'évaluer le potentiel de fertilité des sols sous cotonniers en Côte d'Ivoire et qui pourrait en être une des principales contraintes. Pour ce faire, un total de 500 parcelles de producteurs de coton dans 5 régions du bassin cotonnier a été choisi pour l'échantillonnage du sol dans l'horizon 0-20 cm. Les analyses chimiques des échantillons de sols au laboratoire ont été réalisées sur les paramètres suivants : granulométrie, pHeau, matière organique, $\mathrm{N}$ total, $\mathrm{P}$ assimilable, $\mathrm{K}, \mathrm{Ca}, \mathrm{Mg}$ et $\mathrm{CEC}$. La caractérisation physique a révélé que les sols étudiés ont une texture essentiellement sableuse dans l'horizon superficiel. Ce qui leur confère une faible capacité de rétention en bases échangeables. La détermination du $\mathrm{pH}$ des sols a montré que le $\mathrm{pH}$ varie de peu acide à neutre $(6,4<\mathrm{pH}<7)$. Les sols possèdent de très faibles teneurs en matière organique total, en azote total, en phosphore disponible (dans les régions Nord, Sud-centre et Ouest), en calcium, en potassium, et de faibles valeurs de la capacité d'échange cationique.

Mots clés : Coton, Sol, Fertilité, Productivité, Côte d’Ivoire

\section{Introduction}

La culture de coton est soumise comme les autres spéculations à plusieurs contraintes, notamment une pluviométrie de plus en plus irrégulière et une baisse de la fertilité des sols. Les causes de la baisse de la fertilité des sols en zone cotonnière de Côte d'Ivoire sont, entre autres, la surexploitation des terres, la pression foncière, les pratiques culturales inadaptées (Zagbaï et al., 2006).

La disparition des jachères de longue durée, entraîne également une dégradation de l'environnement de production. Sur le long terme, il y a donc une tendance à la baisse des rendements (N'Goran et al., 2009 ; Intercoton, 2010).

En outre, les doses d'engrais $(200 \mathrm{~kg} / \mathrm{ha})$ et formules d'engrais $(15 \mathrm{~N}$ $15 \mathrm{P} 15 \mathrm{~K}+6 \mathrm{~S}+1 \mathrm{~B})$ vulgarisées actuellement dans la zone cotonnière ont été mises au point depuis une trentaine d'années (Bouchy, 1970 ; Latham, 1971 ; Deat, 1975 ; Sement, 1983). Dans cet intervalle de temps, il est très probable qu'il y ait eu des modifications dans les équilibres chimiques des éléments 
minéraux des sols cultivés avec apparition des carences nouvelles dans les régions cotonnières.

L'évaluation de ces contraintes (baisse des teneurs en éléments minéraux et apparition de nouvelles carences) constitue une étape indispensable qui permettra d'identifier les techniques appropriées de gestion efficiente de la fertilité des sols dans les systèmes de culture à base de cotonniers pour une production durable dans le bassin cotonnier.

En outre, depuis les travaux pionniers de Fritz et Vallerie (1971) et de Braud (1973), la recherche cotonnière en Côte d'Ivoire en matière de fertilisation a très peu évolué. Peu d'information existe sur les caractéristiques édaphiques (physiques, chimiques et biologiques) des différentes zones de production cotonnière. Or, les paramètres précités jouent un rôle majeur dans les techniques spécifiques de fertilisation des sols pour accroître le rendement des cultures. Dans le système de culture actuel, les rendements sont encore faibles. Ils sont estimés à environ $1469 \mathrm{~kg} / \mathrm{ha}$ (Chiapo, 2001). C'est dans ce contexte que cette étude a été initiée en vue de caractériser les sols des cinq (5) principales régions de production du bassin cotonnier de Côte d'Ivoire, d'en déterminer leur état de fertilité et d'identifier les principales contraintes physiques et chimiques susceptibles de limiter la production cotonnière.

\section{Matériel et méthodes :}

\section{Cadre de l'étude}

L'étude a été conduite dans les cinq grandes zones de production du bassin cotonnier à savoir : le Nord-ouest (Odienné, Tengrela et Boundiali) ; le Nord (Korhogo et Ferkessédougou) ; le Centre (Mankono, Katiola et Bouaké) ; l'Ouest (Séguela et Touba) et le Sud (Bouaflé, Zuénoula et Vavoua) (Figure 1). La zone cotonnière ivoirienne est caractérisée par un climat tropical. Deux régimes pluviométriques (monomodal et bimodal) caractérisent cette zone avec des précipitations moyennes oscillant entre 895 et $1197 \mathrm{~mm}$ par an (Kouamé, 1992). La végétation de la zone cotonnière est subdivisée en deux grands types de paysages, à savoir le paysage forestier correspondant au Centre-Ouest et l'Est appartenant au domaine guinéen et le paysage de savane correspondant à la partie Nord de la zone cotonnière qui appartient au domaine soudanais. 


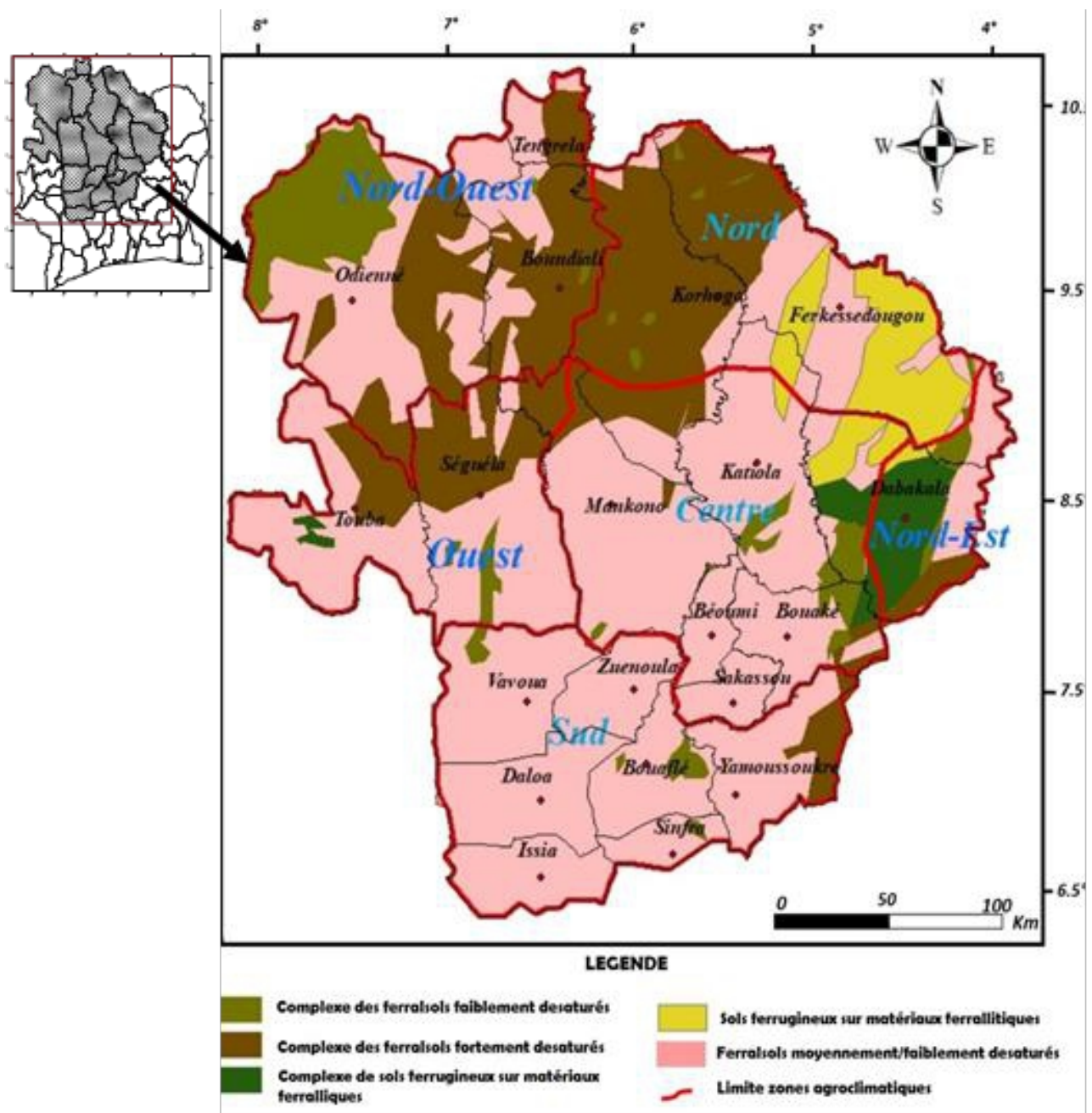

Figure1 : Cinq grandes régions du bassin cotonnier de Côte d'Ivoire

Entre ces deux domaines, il existe une zone de transition nommé zone de contact forêt-savane correspondant au Centre de la zone cotonnière caractérisée par la présence de lambeaux de forêt mésophile, et de larges mailles de savanes séparées par des îlots de forêts galeries (Brou, 2005). La zone cotonnière est essentiellement constituée de ferrasols moyennement et fortement désaturés (Dekoula, et al., 2014; FAO, 2014).

\section{Choix des parcelles}

Dans chacune des cinq régions de la zone cotonnière selon le découpage agro-climatique de la SODEXAM (2008), 10 localités ont été retenues pour la présente investigation. Dans l'optique de couvrir au maximum chaque zone agro-climatique du bassin cotonnier, 10 exploitations ont été choisies par localité, soit 100 exploitations cotonnières par localité. Le dispositif a 
également tenu compte des 4 orientations cardinales (Nord, Sud, Est et Ouest) pour le choix des 10 exploitations.

\section{Dispositif expérimental et méthode de prélèvement des échantillons de sols}

L'échantillonnage des sols a été effectué selon un dispositif en randomisation totale dans les horizons $0-20 \mathrm{~cm}$, influencés par la matière organique et reconnus comme source d'environ $80 \%$ de l'apport nutritionnel des cultures annuelles telle que le cotonnier (N'guessan et al., 2016). Les échantillons de sols ont été prélevés en 2014 et 2015, à la tarière. Dans chaque exploitation cotonnière, 30 échantillons élémentaires de sol ont été prélevés soit un total de 300 échantillons par localité.

Afin d'obtenir des résultats plus représentatifs de la réalité du sol dans chaque localité, 10 échantillons composites ont été constitués à partir d'un mélange des 30 prises élémentaires équipondérales.

Le conditionnement des échantillons de sols après le prélèvement sur le terrain a été effectué à la station du CNRA à Bouaké. Il a consisté d'abord un séchage à l'air libre, un émottage, un tamisage au tamis de $2 \mathrm{~mm}$ pour recueillir la terre fine et un ensachage des échantillons de sols. Ces échantillons ont été transportés au laboratoire Eaux-Sol-Végétaux de 1'Ecole Supérieure d'Agronomie (ESA) à l'Institut National Polytechnique Félix Houphouët Boigny de Yamoussoukro (INPHB), dont les méthodes sont reconnues.

\section{Analyses au laboratoire}

Les analyses physico-chimiques réalisées selon les méthodes standards (FAO, 1984), ont concerné 11 variables quantitatives : la granulométrie, le $\mathrm{pH}$, le Carbone (C), l'Azote (N), le Phosphore assimilable (P ass.), ( $\mathrm{K}^{+}, \mathrm{Ca}^{2+}$, $\mathrm{Mg}^{2+}$, capacité d'échange cationique (CEC), taux de saturation en bases (V). Le carbone déterminé a été multiplié par 1,724 pour estimer la matière organique sous végétation cultivée (Soltner, 2014).

\section{Analyse statistique}

Toutes les données obtenues ont été soumises à une analyse de variance avec le logiciel SPSS où les comparaisons des moyennes ont été réalisées aux seuils de signification de 0,05 avec le test de Newman-Keul. 


\section{Résultats}

Caractéristiques physico-chimiques des sols des régions du bassin cotonnier

Les résultats portant les caractéristiques physiques des sols des différentes régions sont consignés dans le tableau 2. Les analyses statistiques ont montré un effet région de culture du coton sur ces caractères physicochimiques des sols. (Tableau 1). Ces analyses ont montré que la région Nordouest du bassin cotonnier a des taux d'argile (14,64\%), de limon grossier $(20,36 \%)$ et de limon fin $(14,78 \%)$ significativement élevés par rapport aux autres régions. Concernant le sable grossier, les régions Centre, Nord et Ouest du bassin cotonnier ont des taux significativement élevés que les régions du Sud-Centre et du Nord-ouest. Au niveau du sable fin (SF), la région du Sudcentre a un taux significativement élevé $(39,6 \%)$ par rapport aux autres régions. D'une façon générale, l'analyse granulométrique a montré que les sols étudiés ont une texture essentiellement sableuse dans les horizons superficiels. Hormis la zone Sud-centre du bassin cotonnier, cette texture sableuse est dominée par du sable grossier. Les teneurs en argile et limon sont minimales dans ces horizons superficiels. Cette texture sableuse a été mentionnée par Yoro (2000). Comparativement aux travaux de Yoro (2000), on note une diminution du taux d'argile et aussi apparition de plus de sables grossiers.

Tableau I : Résultats des analyses statistiques

\begin{tabular}{|c|c|c|c|c|c|c|}
\hline & ource & $\begin{array}{l}\text { Carré } \\
\text { moyen }\end{array}$ & $\mathbf{F}$ & $P$ calculé & $\underset{\text { théorique }}{\mathbf{p}}$ & Signification \\
\hline & A & 111,962 & 5,765 & 4,732060862 & , 000 & Ths \\
\hline & $\mathrm{Lg}$ & 643,612 & 22,463 & 11,34558945 &, 000 & Ths \\
\hline & Lf & 123,933 & 3,816 & 4,978614265 & ,006 & Ths \\
\hline & $\mathrm{Sg}$ & 1167,87 & 6,641 & 15,28312795 & ,000 & Ths \\
\hline & Sf & 699,144 & 9,373 & 11,82492283 & ,000 & Ths \\
\hline & pHeau & 1,543 & 8,953 & 0,555517776 & , 000 & Ths \\
\hline & $\operatorname{COT}(\%)$ & 5,949 & 21,82 & 1,090779538 & , 000 & Ths \\
\hline Région & M.O. (\%) & 11,126 & 21,363 & 1,491710428 & ,000 & Ths \\
\hline & $\mathrm{N}(\%)$ & 0,016 & 10,281 & 0,056568542 & ,000 & Ths \\
\hline & $\begin{array}{l}\text { P. ass. } \\
\text { (ppm) }\end{array}$ & 1975,403 & 24,193 & 19,87663452 & ,000 & Ths \\
\hline & $\begin{array}{l}\text { CEC } \\
(\mathrm{cmol} / \mathrm{kg})\end{array}$ & 60,304 & 6,179 & 3,472866251 & ,000 & Ths \\
\hline & $\begin{array}{l}\mathrm{Ca} 2+ \\
(\mathrm{cmol} / \mathrm{kg})\end{array}$ & 9,157 & 9,005 & 1,353292282 & ,000 & Ths \\
\hline & $\begin{array}{l}\mathrm{Mg} 2+ \\
(\mathrm{cmol} / \mathrm{kg})\end{array}$ & 0,615 & 6,496 & 0,350713558 & ,000 & Ths \\
\hline
\end{tabular}




\begin{tabular}{lrrrrr}
\hline $\begin{array}{l}\mathrm{K}+ \\
(\mathrm{cmol} / \mathrm{kg})\end{array}$ & 0,139 & 1,487 & 0,16673332 & 0,209 & Ns \\
\hline $\begin{array}{l}\mathrm{Na}+ \\
(\mathrm{cmol} / \mathrm{kg})\end{array}$ & 0,024 & 3,827 & 0,069282032 & 0,005 & Ths \\
\hline $\mathrm{C} / \mathrm{N}$ & 607,03 & 612,568 & 11,01843909 &, 000 & Ths \\
\hline $\mathrm{SBE}$ & 13,646 & 7,669 & 1,652029055 &, 000 & $\mathrm{Ths}$ \\
\hline $\mathrm{V}$ & 3476,074 & 18,305 & 26,36692625 &, 000 & $\mathrm{Ths}$ \\
\hline $\mathrm{Mg} / \mathrm{K}$ & 81,676 & 5,167 & 4,041682818 & 0,001 & $\mathrm{Ths}$ \\
\hline $\mathrm{Ca} / \mathrm{Mg}$ & 15,267 & 12,379 & 1,747398066 &, 000 & $\mathrm{Ths}$ \\
\hline $\mathrm{K} / \mathrm{CEC}$ & 32,246 & 2,656 & 2,539527515 & 0,065 & $\mathrm{Ns}$ \\
\hline
\end{tabular}

Ths : très hautement significatif ; Ns : Non significatif

Tableau II : Caractéristiques physiques des sols des régions du bassin cotonnier

\begin{tabular}{llllll}
\hline Régions & $\begin{array}{l}\text { Argile } \\
(\mathbf{A})\end{array}$ & $\begin{array}{l}\text { Limon Grossier } \\
(\mathbf{L G})\end{array}$ & $\begin{array}{l}\text { Limon Fin } \\
(\mathbf{L F})\end{array}$ & $\begin{array}{l}\text { Sable grossier } \\
(\mathbf{S G})\end{array}$ & Sable fin (SF) \\
\hline Nord-ouest & $14,64( \pm 0,90) \mathrm{a}$ & $20,36( \pm 1,09) \mathrm{a}$ & $14,78( \pm 1,16) \mathrm{a}$ & $27,21( \pm 2,70) \mathrm{a}$ & $22,93( \pm 1,76) \mathrm{a}$ \\
\hline Nord & $\begin{array}{l}10,75( \pm 0,61) \\
\mathrm{b}\end{array}$ & $10,54( \pm 0,74) \mathrm{c}$ & $9,77( \pm 0,79) \mathrm{b}$ & $39,22( \pm 1,83) \mathrm{b}$ & $29,71( \pm 1,19) \mathrm{b}$ \\
\hline Centre & $\begin{array}{l}10,77( \pm 0,61) \\
\mathrm{b}\end{array}$ & $8,36( \pm 0,75) \mathrm{c}$ & $10,75( \pm 0,79) \mathrm{ab}$ & $38,64( \pm 1,85) \mathrm{b}$ & $31,46( \pm 1,20) \mathrm{b}$ \\
\hline Sud-centre & $8,14( \pm 1,17) \mathrm{b}$ & $14,81( \pm 1,43) \mathrm{b}$ & $12,750( \pm 1,52) \mathrm{ab}$ & $24,686( \pm 3,54) \mathrm{a}$ & $39,604( \pm 2,30) \mathrm{c}$ \\
\hline Ouest & $\begin{array}{l}10,07( \pm 1,27) \\
\mathrm{b}\end{array}$ & $11,51( \pm 1,54) \mathrm{c}$ & $13,15( \pm 1,64) \mathrm{ab}$ & $39,34( \pm 3,82) \mathrm{b}$ & $25,94( \pm 2,49) \mathrm{ab}$ \\
\hline $\begin{array}{l}\text { Ferralsol } \\
\text { haplic } \\
\text { (yoro, 2000) }\end{array}$ & 21,5 & 7,2 & 11,4 & 24,7 & 28,4 \\
\hline
\end{tabular}

Les moyennes affectées d'une même valeur dans une même colonne de sont pas significativement différentes au seuil $\alpha<0,05$, selon la méthode de Student-Newman-Keuls (SNK).

\section{Statut organique et acido-basique des sols sous cotonniers}

Le tableau 3 présente les résultats du statut organique et acido-basique des 5 régions du bassin cotonnier. Le statut organique et acido-basique constitue un critère du niveau de fertilité des sols. De ce fait, les normes d'interprétation de Landon (1991) et celles de l'INERA (Kambire, 2000), les échelles de fertilité de Dabin (1970), les valeurs seuils proposées par Boyer (1972 ; 1982) pour les sols tropicaux ont été utilisées. 
Tableau III : Statuts organiques, acido-basique des sols des 5 régions du bassin cotonnier de Côte d'Ivoire.

\begin{tabular}{lcccr}
\hline Régions & \multicolumn{1}{c}{ pHeau } & M.O $(\%)$ & Ntot $(\%)$ & C/N \\
\hline Nord-ouest & $6,38( \pm 0,08) \mathrm{a}$ & $1,33( \pm 0,14) \mathrm{a}$ & $0,07( \pm 0,00) \mathrm{a}$ & $10,71( \pm 0,20) \mathrm{a}$ \\
\hline Nord & $6,43( \pm 0,05) \mathrm{a}$ & $1,80( \pm 0,10) \mathrm{b}$ & $0,09( \pm 0,00) \mathrm{ab}$ & $11,06( \pm 0,13) \mathrm{a}$ \\
\hline Centre & $6,51( \pm 0,05) \mathrm{a}$ & $1,03( \pm 0,10) \mathrm{a}$ & $0,09( \pm 0,00) \mathrm{ab}$ & $19,365( \pm 0,13) \mathrm{b}$ \\
\hline Sud-centre & $6,87( \pm 0,11) \mathrm{b}$ & $2,01( \pm 0,19) \mathrm{b}$ & $0,11( \pm 0,01) \mathrm{b}$ & $10,722( \pm 0,28) \mathrm{a}$ \\
\hline Ouest & $7,06( \pm 0,12) \mathrm{b}$ & $2,95( \pm 0,20) \mathrm{c}$ & $0,16( \pm 0,01) \mathrm{c}$ & $10,939( \pm 0,28) \mathrm{a}$ \\
\hline $\begin{array}{l}\text { Ferralsol Haplic } \\
\text { (Yoro, 2000) }\end{array}$ & 6,4 & 3,9 & 1,23 & 18,57 \\
\hline VSM & 5,5 & 2 & 0,2 & $9<\mathrm{C} / \mathrm{N}<12$ \\
\hline
\end{tabular}

Les moyennes affectées d'une même valeur dans une même colonne de sont pas significativement différentes au seuil $\alpha<0,05$, selon la méthode de Student-Newman-Keuls $(\mathrm{SNK}) ; \mathrm{VSM}=$ Valeur seuil moyenne ; $\mathrm{MO}=$ matière organique, Ntot= Azote total $; \mathrm{C} / \mathrm{N}=$ Rapport Carbone/Azote ;

\section{pHeau}

Les pHeau des différents sols étudiés vont de 6,3 à 7. L'analyse statistique a montré un effet région sur la valeur du pH. Selon Baize (2000), ces sols sont des sols peu acides à neutres. Les sols des régions Nord-ouest, Nord et Centre du bassin cotonnier sont peu acides et sont statistiquement différents des sols des régions Sud-centre et Ouest qui ont des pHeau neutres. Ces valeurs de $\mathrm{pH}$ obtenus sont de façon générale supérieures au seuil proposée dans la littérature qui est $\mathrm{pH}<5,5$ (Baize, 2000). Ces résultats comparés aux ceux de Yoro (2000) montre une faible évolution du $\mathrm{pH}$.

\section{Matière organique (MO)}

Les teneurs moyennes en matières organiques enregistrées dans les sols des différentes régions se situent entre $1 \%$ et 3\% (tableau 3). Un effet région a été observé sur le taux de matière organique au niveau des sols cultivés en coton. Trois groupes homogènes se distinguent suite au test statistique. Le premier groupe est constitué des sols des régions Centre et Nord-ouest qui ont enregistré les plus faibles taux de matières organiques. Le second groupe est constitué des régions Nord et Sud-centre qui ont des valeurs intermédiaires. La région de l'Ouest constitue le dernier groupe avec un de taux de matière organique $(2,96 \%)$. Seul ce taux est supérieur à la valeur seuil moyenne proposée par la littérature qui est de 2\% (Dabin et Bouyer 1963). Comparativement aux travaux de Yoro (2000), on note une forte diminution de la matière organique dans tout le bassin cotonnier.

\section{Azote total}

Les taux d'azote dans les différentes régions varient de 0,07 à $0,16 \%$. Il y a eu un effet de la région sur le taux d'azote (tableau 1). Le taux d'azote de la Région Nord-ouest $(0,07 \%)$ a été la plus faible valeur et est 
significativement différente des valeurs obtenues au niveau des régions Sudcentre et Ouest (respectivement $0,11 \%$ et $0,16 \%$.). Tous les sols des différentes régions du bassin cotonnier ivoirien sont carencés en azote avec des teneurs moyennes inférieures à la valeur seuil de l'ordre de 0,2\% (Landon, 1991). On note également une baisse importante de la teneur en azote des sols dans le bassin cotonnier en comparant ces résultats à ceux de Yoro (2000).

\section{Rapport C/N}

Le rapport $\mathrm{C} / \mathrm{N}$ est un indicateur de la dynamique du carbone et de 1'azote. Les valeurs des ratios $\mathrm{C} / \mathrm{N}$ obtenus ont 10,71 à 19 . Les analyses ont montré un effet régions de culture sur ce rapport. Deux grands ensembles homogènes ont été obtenus. Le premier groupe est constitué des régions Nordouest, Sud-centre, ouest et Nord. Le second groupe est constitué de la région centre avec un rapport $\mathrm{C} / \mathrm{N}$ de 19,37. Les valeurs moyennes de $\mathrm{C} / \mathrm{N}$ obtenues dans les sols du premier groupe sont comprises entre 9 et 12. Cela traduit une minéralisation normale de la matière organique dans ces sols des régions Nord-ouest, Nord, Centre et Sud-centre (Landon, 1991 ; Boyer 1982). Dans la région Centre, on a une valeur moyenne de 19,37, ce qui indique une minéralisation lente de la matière organique, c'est-à-dire une fourniture lente d'azote et autres éléments minéraux aux plantes. Il peut donc y avoir risque élevé de manque d'azote. Par rapport aux travaux de Yoro (2000), on note a baissé dans la majeure partie du bassin cotonnier. Les valeurs obtenues sont comprises entre 9 et 12, exceptée la région Centre.

\section{Statut nutritif selon les bases échangeables et le phosphore assimilable}

Les données du tableau 4 donnent les niveaux de de la fertilité des sols selon les valeurs moyennes des bases échangeables et du phosphore assimilable.

\section{Capacité d'échange cationique (CEC) et Bases échangeables}

La CEC représente l'ensemble des cations échangeables dans le sol $\left(\mathrm{Ca}^{2+}, \mathrm{Mg}^{2+}, \mathrm{K}^{+} ; \mathrm{Na}^{+}\right)$. Les valeurs moyennes de la CEC obtenues dans les différentes régions se situent entre 5,74 et $9,56 \mathrm{Cmol}^{+} \mathrm{kg}^{-1}$. Les analyses ont montré un effet région sur les différentes valeurs de la CEC obtenues. Deux groupes homogènes ont été obtenus. Les régions Sud-centre, Nord, Centre constituent le premier groupe. Les régions Nord-ouest et de l'Ouest constituent le deuxième groupe. Les valeurs de CEC des sols des tous les régions prospectées sont inférieures à la valeur seuil qui est de l'ordre de 15 $\mathrm{Cmol}(+) \cdot \mathrm{kg}^{-1}$ proposée dans la littérature (Landon, 1991). Les faibles capacités d'échanges cationiques confèrent à ces sols une faible capacité de rétention des bases $\left(\mathrm{Ca}^{2+}, \mathrm{Mg}^{2+}, \mathrm{K}^{+}\right.$et $\left.\mathrm{Na}^{+}\right)$provenant de leur minéralisation interne et une rétention réduite des cations en cas d'apport d'engrais minéral. 
Par rapport aux travaux de Yoro (2000), on constate une chute des valeurs de la CEC dans tout le bassin cotonnier.

Les valeurs moyennes obtenues de $\mathrm{Ca}^{2+}$ varient de 1,19 à 3,14 $\mathrm{Cmol}+\mathrm{kg}^{-1}$. Les analyses statistiques ont montré un effet région de culture du coton sur les teneurs en Calcium. Les régions Nord-ouest, Nord, Centre ont des teneurs en calcium statistiquement identiques. Les régions Centre et et Sud-centre ont des teneurs en calcium statistiquement équivalentes. La région de l'Ouest a la plus forte teneur en calcium $(3,14 \mathrm{Cmol}+\mathrm{kg}-1)$ et statistiquement différentes des valeurs de toutes les régions. Toutes ces valeurs de calcium obtenues sont inférieures à la valeur seuil proposée dans la littérature (Landon, 1991) qui est de $4 \mathrm{Cmol}+. \mathrm{kg}^{-1}$. Ce qui indique des déficiences en calcium dans les sols du bassin cotonnier. Ces déficiences sont à des degrés divers.

Les teneurs en magnésium des sols des différentes régions varient de 0,62 à $1,11 \mathrm{Cmol}+\mathrm{kg}^{-1}$. Un effet région de culture du coton a été observé sur les teneurs en magnésium. Deux groupes homogènes ont été obtenus. Le premier groupe est constitué des régions Nord, Nord-ouest, Sud-centre et Centre. La région Ouest forme le deuxième groupe. Les différentes teneurs obtenues sont supérieures à la valeur seuil proposée dans la littérature qui est de 0,5 Cmol+. $\mathrm{kg}^{-1}$ (Landon, 1991). Ce qui indique que les sols des différentes régions du bassin cotonnier ne sont pas dépourvus en magnésium.

Les teneurs en potassium des différentes régions varient de 0,10 à 0,25 $\mathrm{Cmol}+\mathrm{kg}^{-1}$. Il n'y pas eu d'effet région de culture sur ces teneurs en potassium. Les sols des régions Nord-ouest, Sud-centre, Centre et Ouest du bassin cotonnier sont déficients en K. Seule la région Nord a un niveau en K qui est supérieur à la valeur seuil proposée par la littérature qui est de 0,2 $\mathrm{Cmol}^{+} . \mathrm{kg}^{-1}$ (Landon, 1991 ; Boyer, 1972).

\section{Taux de saturation en bases}

Le taux de saturation a varié de 23 à 55, $68 \%$. Un effet région de culture a été observé sur les valeurs obtenues suites aux analyses statistiques. Trois groupes homogènes ont été observés. Les régions Nord, Ouest et Sudcentre constituent le premier groupe avec les plus grandes valeurs. La région Centre et Nord-ouest constituent respectivement le deuxième et troisième groupe. Le taux de saturation est un indicateur de la richesse chimique des sols et de la garniture cationique du complexe adsorbant. Les valeurs obtenues révèlent que les taux de saturation sont moyens et les sols étudiés sont en général moins fertiles ( $<$ à 60). Les travaux de Yoro (2000) indiquent que les sols du bassin cotonnier ont toujours été moins saturés ( $\mathrm{V}<60 \%)$. 


\section{Phosphore assimilable}

Les teneurs moyennes en phosphore ont varié de 5,42 à 28,45 ppm. Les analyses statistiques ont montré un effet significatif de région de culture sur les valeurs de phosphore obtenues. Quatre groupes homogènes ont été obtenus. Les régions Sud-centre constitue le premier groupe avec la plus faible valeur $(5,52 \mathrm{ppm})$. Les régions Nord, Centre et Nord-ouest constituent respectivement le deuxième, troisième et quatrième groupe. La région Nordouest possède significativement la plus grande teneur en phosphore 28,45 $\mathrm{ppm})$. Les teneurs moyennes en phosphore assimilable des régions Nord, Sudcentre et Ouest sont en dessous de la valeur seuil qui est de $15 \mathrm{ppm}$ proposée dans la littérature (Landon, 1991). Cela indique une déficience en phosphore dans les sols de ces régions. Les résultats des travaux de Yoro (2000) montrent que les teneurs en phosphore ont fortement chuté dans le bassin cotonnier.

Tableau IV : Statut nutritif des sols des 5 régions du bassin cotonnier de Côte d'Ivoire

\begin{tabular}{|c|c|c|c|c|c|c|c|}
\hline Régions & $\begin{array}{l}\mathbf{P}_{\text {olsen }} \\
\text { (ppm) }\end{array}$ & $\begin{array}{l}\mathrm{T}=\mathrm{CEC} \\
\left(\mathrm{Cmol}^{+} . \mathrm{kg}\right. \\
-1)\end{array}$ & $\begin{array}{l}\text { Caéch } \\
\left(\mathrm{Cmol}^{+} . \mathrm{kg}^{-1}\right)\end{array}$ & $\begin{array}{l}\text { Mgéch } \\
\left(\mathrm{Cmol}^{+} . \mathrm{kg}^{-1}\right)\end{array}$ & $\begin{array}{l}\mathrm{K}_{\text {éch }} \\
\left(\mathrm{Cmmol}^{+} . \mathbf{k}\right. \\
\left.\mathrm{g}^{-1}\right)\end{array}$ & $\begin{array}{l}\mathrm{S} \\
\left(\mathrm{Cmol}^{+} . \mathrm{kg}\right. \\
-1)\end{array}$ & $\begin{array}{c}V \%= \\
S / T * 100\end{array}$ \\
\hline Nord-ouest & $\begin{array}{l}28,45( \pm 1,84) \\
d\end{array}$ & $\begin{array}{l}8,90 \\
( \pm 0,63) b\end{array}$ & $\begin{array}{l}1,19 \\
( \pm 0,20) \mathrm{a}\end{array}$ & $\begin{array}{l}0,68 \\
( \pm 0,06) a\end{array}$ & $\begin{array}{l}0,09 \\
( \pm 0,06)\end{array}$ & $\begin{array}{l}1,97 \\
( \pm 0,27) \mathrm{a}\end{array}$ & $\begin{array}{l}23,02 \\
( \pm 2,81) \mathrm{a}\end{array}$ \\
\hline Nord & $\begin{array}{l}12,61 \\
( \pm 1,25) \mathrm{b}\end{array}$ & $\begin{array}{l}5,99 \\
( \pm 0,43) a\end{array}$ & $\begin{array}{l}1,91 \\
( \pm 0,14) a b\end{array}$ & $\begin{array}{l}0,62 \\
( \pm 0,04) a\end{array}$ & $\begin{array}{l}0,25 \\
( \pm 0,04)\end{array}$ & $\begin{array}{l}2,79 \\
( \pm 0,18) a\end{array}$ & $\begin{array}{l}46,78 \\
( \pm 1,91) \mathrm{c}\end{array}$ \\
\hline Centre & $\begin{array}{l}21,37( \pm 1,26) \\
\mathrm{c}\end{array}$ & $\begin{array}{l}6,89 \\
( \pm 0,43) a\end{array}$ & $\begin{array}{l}1,54 \\
( \pm 0,14) \mathrm{ab}\end{array}$ & $\begin{array}{l}0,74 \\
( \pm 0,04) \mathrm{a}\end{array}$ & $\begin{array}{l}0,14 \\
( \pm 0,04)\end{array}$ & $\begin{array}{l}2,43 \\
( \pm 0,18) \mathrm{a}\end{array}$ & $\begin{array}{l}37,72 \\
( \pm 1,93) \mathrm{b}\end{array}$ \\
\hline Sud-centre & $\begin{array}{l}5,42( \pm 2,41) \\
\mathrm{a}\end{array}$ & $\begin{array}{l}5,74 \\
( \pm 0,83) \text { a }\end{array}$ & $\begin{array}{l}2,18 \\
( \pm 0,29) \mathrm{b}\end{array}$ & $\begin{array}{l}0,72 \\
( \pm 0,08) \mathrm{a}\end{array}$ & $\begin{array}{l}0,11 \\
( \pm 0,8)\end{array}$ & $\begin{array}{l}3,02 \\
( \pm 0,38) \mathrm{a}\end{array}$ & $\begin{array}{l}55,68 \\
( \pm 3,68) \mathrm{c}\end{array}$ \\
\hline Ouest & $\begin{array}{l}8,33( \pm 2,60) \\
a b\end{array}$ & $\begin{array}{l}9,56 \\
( \pm 0,90) \mathrm{b}\end{array}$ & $\begin{array}{l}3,14 \\
( \pm 0,29) \mathrm{c}\end{array}$ & $\begin{array}{l}1,11( \pm 0,08) \\
b\end{array}$ & $\begin{array}{l}0,18 \\
( \pm 0,08)\end{array}$ & $\begin{array}{l}4,44 \\
( \pm 0,38) b\end{array}$ & $\begin{array}{l}49,36 \\
( \pm 3,97) \mathrm{c}\end{array}$ \\
\hline $\begin{array}{l}\text { Ferralsol } \\
\text { Haplic } \\
\text { (Yoro, } \\
2000 \text { ) }\end{array}$ & 37 & 13,71 & - & - & - & 6,66 & 48,58 \\
\hline VSM & 15 & 15 & 4 & 0,5 & 0,2 & 3 & 60 \\
\hline
\end{tabular}

Les moyennes affectées d'une même valeur dans une même colonne de sont pas significativement différentes au seuil $\alpha<0,05$, selon la méthode de Student-Newman-Keuls $(\mathrm{SNK}) ; \mathrm{VSM}=$ Valeur seuil moyenne $; \mathrm{P}=$ phosphore assimilable $; \mathrm{CEC}=$ Capacité d'échange cationique $; \mathrm{Ca}=\mathrm{Calcium}$ échangeable ; $\mathrm{Mg}=$ Magnésium échangeable ; $\mathrm{K}=$ Potassium échangeable ; $\mathrm{SBE}=$ Somme des bases échangeables ; V=Taux de saturation.

\section{Statut nutritif évalué selon les cas des équilibres chimiques}

Pour diagnostiquer les équilibres minéraux et juger de la carence relative des bases échangeables dans les sols du bassin cotonnier de Côte d'Ivoire, les valeurs seuils convenables sont celles établies pour le cotonnier et spécifiquement pour la Côte d'Ivoire. Lorsque des valeurs spécifiques n'existent pas, des valeurs seuils pour les sols tropicaux en général ont été mises à contribution. Le tableau 5 présente les caractéristiques des équilibres chimiques des sols des différentes régions du bassin cotonnier de Côte d'Ivoire. 
Tableau V : Caractéristiques des équilibres chimiques des sols des différentes régions du bassin cotonnier de Côte d'Ivoire

\begin{tabular}{lccc}
\hline Régions & $\mathbf{M g} / \mathbf{K}$ & $\mathbf{C a} / \mathbf{M g}$ & K/CEC $(\%)$ \\
\hline Nord-ouest & $8,77( \pm 0,81) \mathrm{b}$ & $1,74( \pm 0,22) \mathrm{a}$ & $1,37( \pm 0,71)$ \\
\hline Nord & $4,69( \pm 0,55) \mathrm{a}$ & $3,30( \pm 0,15) \mathrm{b}$ & $3,86( \pm 0,48)$ \\
\hline Centre & $6,35( \pm 0,55) \mathrm{ab}$ & $2,06( \pm 0,15) \mathrm{a}$ & $2,37( \pm 0,48)$ \\
\hline Sud-centre & $7,36( \pm 1,06) \mathrm{ab}$ & $3,00( \pm 0,29) \mathrm{b}$ & $2,07( \pm 0,93)$ \\
\hline Ouest & $7,90( \pm 1,14) \mathrm{b}$ & $2,79( \pm 0,32) \mathrm{b}$ & $1,97( \pm 1,00)$ \\
\hline VSM & $3<\mathrm{Mg} / \mathrm{K}<25$ & $3<\mathrm{Ca} / \mathrm{Mg}<5$ & K/CEC $\geq 2 \%$ \\
\hline
\end{tabular}

Les moyennes affectées d'une même valeur dans une même colonne de sont pas significativement différentes au seuil $\alpha<0,05$, selon la méthode de Student-Newman-Keuls (SNK) ; VSM= Valeur seuil moyenne ; $\mathrm{Mg} / \mathrm{K}=$ Rapport Magnésium/Potassium ; $\mathrm{Ca} / \mathrm{Mg}=$ Rapport Calcium/Magnésium ; K/CEC = Taux de saturation du potassium.

\section{Equilibre $\mathrm{Mg} / \mathrm{K}$}

Le tableau 5 indique les rapports $\mathrm{Mg} / \mathrm{K}$ dans les différents sols. Ces rapports $\mathrm{Mg} / \mathrm{K}$ sont compris entre 4,6 et 8,77. Les analyses statistiques ont montré un effet significatif de la région de culture sur le rapport $\mathrm{Mg} / \mathrm{K}$. Deux groupes homogènes distincts ont été obtenus. Le premier groupe est formé par la région Nord avec un rapport faible (4,69). Le deuxième groupe est constitué des Nord-ouest et Ouest avec des rapports significavement élevés. Pour ces deux cations reconnus comme antagonistes, les valeurs optimums du rapport $\mathrm{Mg} / \mathrm{K}$ se situent entre 3 et 25 pour le cotonnier en côte d'Ivoire (Fritz et Vallerie, 1971 ; Boyer, 1982). Pour la région Nord, on constate un rapport avec une valeur(4,69) qui tend vers la limite inférieure du rapport $\mathrm{Mg} / \mathrm{K}$ qui est de 3 pour le cotonnier. Cela indique que des déficiences en magnésium peuvent surgir surtout dans la région Nord qui est une grande région de production de coton.

\section{Equilibre $\mathbf{C a} / \mathbf{M g}$}

Les valeurs du rapport $\mathrm{Ca} / \mathrm{Mg}$ obtenues dans les différentes régions du bassin cotonnier ont varié de 1,74 à 3,1 (tableau 5). Les analyses statistiques ont indiqué qu'il y a une différence significative entre les valeurs obtenues dans les différentes régions. Deux groupes homogènes ont été obtenus. Les régions Nord-ouest et Centre ont de faibles valeurs et constituent le premier groupe. Les régions Nord, Sud-centre et Ouest constituent le deuxième groupe. Les valeurs optimales du rapport $\mathrm{Ca} / \mathrm{Mg}$ sont comprises entre 3 et 5 selon Landon (1991). Pour les régions Nord-ouest et Centre, on observe des valeurs inférieures à 3 . Ce qui indique une possible inhibition du $\mathrm{P}$ et une déficience du Calcium dans toutes les régions du bassin cotonnier. Toutes les régions du bassin cotonnier sont à surveiller car toutes les valeurs du rapport sont autour de 3 qui la valeur seuil à ne pas franchir. 


\section{$\mathrm{K} / \mathrm{CEC}(\%)$}

Les valeurs des pourcentages de saturation du Potassium (K) dans les différentes régions vont de 1,37 à $3,86 \%$. Les analyses statistiques n'ont pas montré de différence significative entre les valeurs des différentes régions. Ces pourcentages sont inférieurs ou égaux au pourcentage seuil qui est de $2 \%$ pour les régions Nord-Ouest, Ouest et Sud-centre. Ces valeurs traduisent une déficience de sols en Potassium dans ces sols. Dans les régions Nord et Centre, les taux obtenus sont compris dans la fourchette optimale $(2<\mathrm{K} / \mathrm{CEC}<5 \%)$.

\section{Discussion}

Les couches supérieures des sols des différentes régions ont une texture sableuse. Cette texture sableuse des sols confère à ces sols un bon drainage, une bonne circulation de l'air et une pénétration facile des racines. Mais cet état a des inconvénients. Ces sols ont une faible capacité de rétention en eau (Mulaji et al., 2016). Les teneurs en argile sont minimales dans ces horizons superficiels (entre 8 et $15 \%$ ). Ce faible taux d'argile n'est sans inconvénients sur les sols étudiés (Baize, 2000). Selon cet auteur l'argile est bien la fraction granulométrique la plus active car elle a de multiples fonctions (association avec la matière organique, la cohésion des agrégats, la fixation des cations et anions sur les sites d'échange, la rétention de l'eau etc.). En effet, cette faible teneur en argile rend ces sols moins fertiles. Pour les faibles teneurs en argiles $(<20 \%)$, il faut davantage de matière organique pour compenser le déficit en colloïdes.

Le $\mathrm{pH}$ du sol détermine le type d'activité (acide ou basique) existante ou prédominante. Les valeurs de $\mathrm{pH}$ obtenues dans les différentes régions sont supérieures au seuil $(\mathrm{pH}>5,5)$ et sont peu acide à neutre. Cela indique que les réactions chimiques (biodisponibilité des éléments nutritifs) et microbiologiques dans ces sols se déroulent convenablement. Généralement, la plupart des plantes cultivées se développent harmonieusement dans un sol neutre ou légèrement acide c'est-à-dire de 5,5 <pH<7 (Landon, 1991 et Baize, 2000). Les faibles valeurs de $\mathrm{pH}$ dans les sols limitent la croissance végétale par la diminution de la nitrification, la déficience en phosphore, la toxicité aluminique et manganique, et par la grande disponibilité des certains éléments mineurs.

La matière organique participe à l'amélioration de la fertilité des sols. Elle est un bon indicateur pour la bonne santé des plantes. Or, dans le cas de cette étude, les valeurs trouvées sont faibles, ce qui pourrait constituer un obstacle à la bonne productivité des cotonniers. Cela pourrait s'expliquer par les pratiques agricoles faites dans les différentes régions du bassin cotonnier que sont la surexploitation des sols et les amendements organiques et chimiques insuffisants (Baize, 2000 ; Sawadogo, 2006 ; N'goran et al., 2015). Cette situation de faible teneur en matière organique expose ces sols à la 
dégradation par l'érosion hydrique lors des fortes précipitations en milieu tropical humide (Mulaji et al., 2016).

La CEC est un autre indicateur de la fertilité potentielle des sols. La capacité d'échange cationique du sol représente la taille du réservoir permettant de stocker de manière réversible certains éléments fertilisants cationiques (potassium, magnésium, calcium...). Elle est liée au complexe argilo humique. La valeur de la CEC d'un sol est fonction des quantités d'argile et de matière organique qu'il contient, mais aussi de la nature des ces éléments et du $\mathrm{pH}$ du sol. Les valeurs moyennes de CEC obtenue sur l'ensemble des sites sont généralement faibles. Elles sont nettement inférieures au seuil tel que décrit par Sawadogo (2006). Cela pourrait s'expliquer par les faibles teneurs en argiles et en matière organique (Baize, 2000). En effet, selon cet auteur, les matières organiques des sols jouent un rôle majeur aussi bien au plan agronomique qu'au plan environnemental, à savoir l'adsorption et la rétention de l'eau, des bases échangeables, du phosphore, de l'azote et des éléments traces métalliques. Les valeurs faibles des CEC des sols ne leur confèrent pas un pouvoir tampon élevé (Baize, 2000). Cela serait défavorable à une nutrition minérale efficiente du cotonnier dans ces zones de production.

Concernant les bases échangeables ( $\mathrm{Ca}, \mathrm{Mg}$ et $\mathrm{K}$ ) pour l'ensemble des sols étudiés, hormis les valeurs trouvées au niveau de $\mathrm{Mg}$, les valeurs obtenues sont inférieures aux valeurs seuils déterminées pour les sols tropicaux (Landon, 1991 ; Boyer, 1982).

Le taux de saturation du complexe adsorbant par les trois cations alcalins et alcalino-terreux est un précieux indicateur pédologique et agronomique quant à la richesse chimique du sol, laquelle détermine l'activité biologique, la qualité de la structure, les réserves en éléments fertilisants. Les taux de saturation moyens obtenus indiquent que les sols sont moins fertiles. Ces états des sols sous cotonniers pourraient s'expliquer par les pratiques agricoles faites dans les différentes régions du bassin cotonnier que sont la surexploitation des sols (la monoculture de coton sur la même parcelle sans rotation ni jachère) et les amendements organiques et chimiques insuffisants (N'goran et $a l ., 2015$; Fagaye et al., 2015). Ces valeurs faibles obtenues au niveau des bases échangeables ne sont pas sans inconvénients sur la nutrition minérale du cotonnier suite au déséquilibre qui existerait au niveau des trois cations essentiels (Mulaji et al., 2016). Donc ces sols sous cotonniers pourraient connaître des problèmes de carence au niveau du potassium.

\section{Conclusion}

Les niveaux des caractéristiques physico-chimiques des sols étudiés permettent d'identifier les principales contraintes à la productivité des sols dans les cinq régions du bassin cotonnier de Côte d'ivoire. 
Les analyses statistiques ont montré des différences significatives entre les différentes régions pour la majorité des variables (Argile, limon, sable, pHeau, Carbone organique total, la matière organique, l'azote, le potassium assimilable, la CEC, la calcium, le magnésium, le rapport $\mathrm{C} / \mathrm{N}$, la somme des bases échangeables, le taux de saturation, les $\mathrm{Mg} / \mathrm{K}, \mathrm{Ca} / \mathrm{Mg}$ ), exception faite pour le potassium $\left(\mathrm{K}^{+}\right)$et le ratio $\mathrm{K} / \mathrm{CEC}$.

La mésures des paramètres physiques a révélé que les sols étudiés ont une texture essentiellement sableuse dans l'horizon superficiel. Ce qui leur confère une capacité de rétention très faible en éléments minéraux apportés par fertilisation

La mesure des paramètres chimiques des sols a montré que ces sols présentent des réactions qui vont de peu acide à neutre $(6,4<\mathrm{pH}<7)$ et possèdent de très faibles teneurs en matière organique total, en azote total, en phosphore disponible (dans les régions Nord, Sud-centre et Ouest), en calcium, en potassium, et de faibles valeurs de la capacité d'échange cationique.

Suite aux contraintes susmentionnées, pour accroitre la production agricole de ces sols, il devient nécessaire de relever leur fertilité chimique et biologique jusqu'au niveau optimal en utilisant des techniques appropriées de gestion. Ces solutions durables impliquent l'utilisation fréquente d'une part d'amendements organiques tels que les fumiers, du compost et les résidus de récolte, et d'autre part la combinaison d'amendements organiques avec les engrais chimiques. L'apport de ces amendements organiques amélioraient la stabilité, la structure et la rétention d'eau du sol et influent sur la chimie en corrigeant l'acidification par l'effet de son pouvoir tampon et en fournissant les substances nutritives progressivement assimilables par les cultures.

\section{References:}

1. Baize, D. (2000). Guide des analyses en pédologie, 2nd Ed. France, INRA Editions. $257 \mathrm{P}$.

2. Bouchy, C. (1970). Contribution à l'étude des déficiences minérales en culture cotonnière de Côte d'Ivoire, Coton et Fibres Tropicales. Vol.25, No. 2, pp. 235-251.

3. Bouyer, S. \& Dabin, B. (1963). Etudes pédologiques du delta central du Niger, Agronomie Tropicale, No 12, pp. 1300-1304.

4. Boyer, J. (1972). Soil Potassium. In soils of the humid tropics; 102135, National Academy of Science publ. (Washington).

5. Boyer, J. (1982). Les sols ferrallitiques, Tome X : facteurs de fertilité et utilisation des sols, Initiations - Documentations Techniques, No 52, ORSTOM, Paris, France.

6. Braud, M. (1973). Le diagnostic foliaire et la nutrition potassique du cotonnier, In Institut International de la Potasse (Eds), le potassium 
dans les cultures et las sols tropicaux. Compte rendu du 10ème colloque organisé en décembre à Abidjan république de Côte d'Ivoire, pp. 265-276.

7. Brou, T. Y. (2005). Climat, mutations socio-économiques et paysages en Côte d'Ivoire. HDR, Université de Lille, 212 p.XI, pp 221- 237. ORSTOM - France.

8. Chiapo A. C. (2001). Analyse de la filière coton dans le contexte de la libéralisation : cas de la zone de Korhogo (Côte d'Ivoire). Ecole Supérieure d'Agronomie (ESA)-INPHB-Diplôme d'Agronomie Approfondie 2001; 77 p.

9. Dabin B. (1970). Les facteurs chimiques de la fertilité des sols (bases échangeables ; sels ; Utilisation des échelles de fertilité) - chapitre XI, pp 221- 237. ORSTOM - France.

10. Deat, M. (1975). Le redressement de la fertilité sur des terres à vocation cotonnière présentant des carences en éléments majeurs", Coton et Fibres Tropicales, Vol. 30, No. 2, pp. 245-262.

11. Dekoula C. S., Kouame B., N'goran E. K., Yao, F. G., Ehounou J. N., et Soro, N. (2018). Impact de la variabilité pluviométrique sur la saison culturale dans la zone de production cotonnière en Côte d'Ivoire. European Scientific Journal, ESJ, Vol 14 No12 : pp.143156.

12. Fagaye, S., Coulibaly, D., Cissé, O., Patrick, D. (2015). Evaluation de l'arrière effet de la culture du coton sur la production céréalière en zone cotonnière du Mali, In M., Fok, N. Ousmane et K. Siaka (Eds.), $A G R A R-2013$ : 1re conférence de la recherche africaine sur l'agriculture, l'alimentation et la nutrition, Yamoussoukro : L'agriculture face aux défis de l'alimentation et de la nutrition en Afrique : quels apports de la recherche dans les pays cotonniers, pp. 149-160.

13. FAO (1984). Méthodes d'analyse physique et chimique des sols et des eaux, Bullettin pédologique de FAO N 10 Rome, 280 p, 1984.

14. FAO (2014). Word Reference Base for Soil Resources. Food and Agriculture

15. Organization of the United Nations, Rome.

16. Fritz, A. \& Vallerie, M. (1971). Contribution à l'étude des déficiences minérales des sols sous culture cotonnière au Nord-Cameroun." Coton et fibres tropicales, Vol 26, No.3, pp. 273-301.

17. Intercoton (2010). La filière coton en Côte d'Ivoire - Importance et difficultés www.intercoton.org, 31p.

18. Kambire F. (2000). Effets des techniques d'utilisation de la Dent IR.12 sur la production du coton et du maïs en zone cotonnière ouest du 
Burkina-Faso, Mémoire de fin d'études, Diplômes d'ingénieur du développement rural, option agronomie, $117 \mathrm{p}$.

19. Kouamé, B. (1992). Adéquation de différentes modèles globaux pluiedébit pour déterminer les apports en eau dans les zones de transition et de forêt de la Côte d'Ivoire : Essai de régionalisation des paramètres. Thèse de doctorat, P.200.

20. Landon, J. R. (1991). Booker Tropical Soil Manual, A handbook for soil survey and agricultural land evaluation in the tropics and subtropics, paperback, longman, Booker Tate limited, Oxon, Royaume Uni, p 474.

21. Latham, M. (1971). Rôle du facteur sol dans le développement du cotonnier en Côte d'Ivoire, Cahier ORSTOM, séries Pédologie, Vol.9, No 1, pp. 29-42.

22. Mulaji, C. Disa-Disa, P., Kibal, I. \& Culot, M. (2016). Diagnostic de l'état agropédologique des sols acides de la province de Kinshasa en république démocratique du Congo (RDC), Comptes rendus Chimie, http://dx.doi.org/10.1016/j.cri.2015.08.010.

23. N'guessan, K. J-C., Akotto, O. F., Snoeck, D., Camara, M., \& YaoKouamé, A. (2016). Potentiel de fertilité des vergers de cacaoyer Thebroma cacao L. (Malvacea) en Côte d'Ivoire. International Journal of Innovation and Applied Studies, IJIAS, Vol. 13 No 3, pp. 868-879.

24. N'goran, K. E., N'guessan, E., Tehia, K. E., Zohouri, G. P. \& Ochou, O. G. (2015). Valorisation de la fumure organique dans les systèmes de culture à base de cotonniers et sécurité alimentaire en Côte d'Ivoire, In M., Fok, N. Ousmane et K. Siaka (Eds.), AGRAR-2013 : 1re conférence de la recherche africaine sur l'agriculture, l'alimentation et la nutrition, Yamoussoukro : L'agriculture face aux défis de l'alimenation et de la nutrition en Afrique : quels apports de la recherche dans les pays cotonniers, pp. 187-195.

25. Sawadogo, H. (2006). Fertilisation organique et phosphaté en système de culture Zaï en milieu soudano-sahelien du Burkina Faso. Thèse de doctorat, faculté universitaire des sciences agronomiques de Gembloux, Belgique, P.219.

26. Sement, G. (1983). La fertilité des systèmes culturaux à base de cotonnier en Côte d'Ivoire. Neuf années d'expérimentation et d'observations multilocales (1973-1982), $40 \mathrm{p}$.

27. Sery, Z. H. (2005). Evaluation de l'impact du coton sur le developpement rural : cas de la région de Korhogo au Nord de la Côte d'Ivoire. Thèse de doctorat, faculté universitaire des sciences agronomiques de Gembloux, Belgique, P. 267.

28. SODEXAM (2008). Découpage agro climatique, Côte d'Ivoire. 
29. Soltner D. (2014). Les bases de la production végétale: Tome 1. Le sol et son amélioration. Collections Sciences et Techniques Agricoles Tome I, $26^{\mathrm{e}}$ édition, Paris, $472 \mathrm{p}$.

30. Yoro G. R. (2000). Les principaux sols de Côte d'Ivoire et leur corrélation avec les groupes de référence de la base mondiale de données des sols (WRB). Quatorzième réunion du sous-comité Ouest et Centre Africain de Corrélation des Sols, 9-13 Octobre 2000, Cotonou 19p.

31. Zagbaï, H.S., Berti, F. \& Lebailly, P. (2006). Impact de la dynamique cotonnière sur le développement rural. Etude de cas de la région de Korhogo, au nord et au centre de la Côte d'Ivoire", Biotecnol.Agron. Soc. Environ. Vol.10, No. 4, pp. 325-334. 